\title{
Analysis of Characteristic Between-Class Exercise Choreography in Primary School under the Background of Tibetan Dance Inheritance in Ganzi
}

\section{- A Case Study of Characteristic Between-class Exercise of Boarding Primary School in Guza District}

Lihan Fan

Sichuan Minzu College

Kangding, China

\begin{abstract}
The characteristic between-class exercise of primary and secondary schools requires movements, and the arrangement of music cater to the theme characteristics, which not only needs to meet the requirements of sports, but also has the role of assisting the cultivation of campus culture. By inheriting Tibetan dance in Ganzi, folk dance and music elements are applied to the choreography of between-class exercise, which meets the development requirements of characteristic exercise. In this context, by summarizing the characteristics of Tibetan folk music and dance in Ganzi, the symbolized elements were selected to carry out the transformation and creation of gymnastics, and primary school students were selected for practical research. At present, the characteristic between-class exercises have been popularized in Yutong district of Ganzi, and some achievements have been achieved.
\end{abstract}

Keywords-between-class exercise; choreograph; characteristic; national culture; inherit

\section{INTRODUCTION}

Under the new situation of the National Basic Education, the fundamental education in Ganzi Tibet has been further improved, especially in hardware and equipment. However, the learning atmosphere in school still remains as a headache. Most schools hang boards write motifs, inspirational stories and local customs and practices on the wall, in a bid to exert an imperceptible influence on students, but it is hard to reap the desired fruits due to the lack of practicality.

Extract Ganzi Tibetan school culture characteristics, such as the regional customs to carry out long-term and stable education in some specific activities in a form popular with primary school students, then the purpose of local folk heritage and construction of campus characteristic culture can be played. Taking into consideration the Tibetan dance inheritance, it can achieve the purpose of building campus characteristic culture by persisting in carrying out between-class exercises.

The author was entrusted by the boarding primary school in Guzá district, Ganzi prefecture to develop a set of characteristics "Tibetan dance" exercise within 3 months. At the beginning, the author assembled a team to sum up the Tibetan folk investigation experience for many years, and try to choreograph Tibetan dance movement. Despite difficulties, the characteristic between-class exercise came out in accordance

This paper is the phased achievement of Musicology, an applicationoriented demonstration major of universities in Sichuan Province. with the requirements in a short period and is applied in Guzá primary school. At present, it has become the fixed activities between classes in the school. This paper analyzes the choreography of elementary school characteristic betweenclass exercise under Guzá Tibetan dance heritage.

\section{BACKGROUND AND REQUIREMENTS OF CHOREOGRAPHY}

Between-class exercise is an important means of physical training for pupils in primary and secondary school education. With the development and change of the times, the demand of between-class exercise is not only to meet the physical fitness requirement, but also to be related to the construction of aesthetics and school culture. In Ganzi Tibetan area, combining the unique rhythm of folk dance with the creation of betweenclass exercise can improve pupils' mental health and increase the effect of aesthetic education. Besides, the good characteristic between-class exercise activity can become the breakthrough of quality education. Pupils are on the key period for the development of intelligence and physical quality. In this stage, long-term, regular and systematic extracurricular activities can enhance pupils' physical and mental health. The integration of Tibetan folk dance elements in the between-class exercise is conducive to pupils' body coordination training, aesthetic training, and the inheritance of traditional culture, etc, and it is also beneficial to the establishment of unique campus culture.

To create and compile characteristic between-class exercise, we should first obtain relevant knowledge and then summarize the local folk music and corresponding dance movements. Then, we should transform the dance movements to the between-class exercise based on relevant PE knowledge. In the course of transformation, we should on one hand grasp the timing, the distribution of exercise intensity and the configuration of music, on the other hand, we should conduct interviews and surveys at all levels, which are the necessary conditions for the development of characteristic between-class exercise.

Besides, the creation and arrangement of characteristic between-class exercise should also follow such principles as scientific exercise, safe implementation and easy learning [1] and interesting. 
Scientific exercise. First, the exercise should meet the time requirements for exercise between classes, that is, it takes the primary pupils less time to have adequate exercise, because too much exercise can be a burden to the body and may affect the teaching quality of the following class. Then, the exercise should meet the law of physical and mental development of pupils, that is, the pupils can take fully exercise and coordinate physical development through such activity. In accordance with the above scientific exercise requirements, the range of movements should be from small to large, the rhythm speed from slow to fast and the physical training from part to the whole. We should ensure that the heart rate of pupils can meet the low - high - low change rule.

Safe implementation. Student safety is the foundation of all school education. During the exercises, accidents should be avoided as far as possible. We should consider the bearing capacity and unexpected accidents that may occur during the completion process. Factors such as the surrounding environment and the limitations of unit density on the body design of pupils in the queue should also be considered thoroughly.

Easy learning and interesting: the whole movement should easy to learn and interesting without burden and let students' body and mentality get relaxed. Through long-term implicit guidance, the traditional elements of Tibetan ethnic groups will be passed on to achieve the common improvement of physical and cultural quality.

\section{ANALYSIS OF INHERITANCE ELEMENTS}

The performance forms of Tibetan folk dances can be divided into five categories, that is, "Dui Xie", "Kang Xie", "Guo Xie", "Qiang Mu" and "Guo Zhuo". Gestures can be summarized into Shuai (tossing), You (swing), La (pulling), Rao (circling), Tui (pushing), Yang (lifting) and Sheng (rising). Legs and feet can be summarized as seven basic elements: Ta (stepping), Mo (grinding), Ca (rubbing), Tuo (dragging), Ti (kicking), Jiao Cha (crossing), Pao (digging) etc [2]. At present, the main categories of Tibetan dance include Guzhuang dance, String dance, Tap dance and Reba dance. The basic characteristics of Tibetan dance can be summarized as follows: the vibration of knee joint with rhythm and the coordination changes of the same hand and foot.

Guozhuang dance is the most common group dance in Tibetan areas. Although different regions have their own music and dance forms, the movements of Guozhuang dance come from life in general, which can be summarized as: stepping, turning, swinging arms and small jumps.

Guozhuang dance can be divided into two categories based on the speed, the one with slow rhythm and smooth and graceful dance, the other with rapid pace and enthusiastic and unrestrained dance. In this two Guozhuang dances, the hands and feet are always in a one-way posture, which requires particularly high coordination. For example, after stepping forward, the dancers often turn over to the left or right with their hands and feet on the same side. In traditional Guozhuang costumes, dancers will wave their long sleeves when they stretch their arms, especially in the Guozhuang dance with fast speed. In the case of Zhenda Guozhuang, the dancers wave and swing their long sleeves forcefully according to the rhythm, and the visual effect is very shocking. These combinations of twists, turns, bends and squats help improve coordination, flexibility and joint flexibility of the body. To sum up, Guozhuang dance is suitable for body rotation, whole body movement, organizing movement and the connection of different periods of primary school exercise.

String dance, like Guzhuang, is a very common group dance in Tibetan areas. The difference is that it gets its name from the accompaniment of stringed instruments. In the display of traditional string dance, string musicians are not only accompaniment members, but also leaders. Under the guidance of the string musicians, the whole team of male dancers is in front, female dancers are behind, the elders are in front, and the young ones are behind, dancing in a clockwise circle. The string dance is performed while singing. It is light and melodious. Batang string dance enjoys a good reputation in Tibetan areas and "three steps a lift, one step a rely [3]" is the summary of its characteristics.

The movements often used in the string dance can be summarized as follows: draw an arc alternately with hands on the chest, step forward with the rhythm of a few steps and then step back; upper body raise sleeve and lower body with side kick; upper body one hand in the waist, one hand up to raise, lower body with the same side stamp feet; alternately arc your hands as you circle forward. According to the style of string music, string dance also include body turns, bends and squats and small amplitude jumps. With the body routine, the string dance is repeated according to the rhythm until the end of the music. In summary, string dance is suitable for stretching, chest expanding and side movement.

As for the tap dance, the main feature is leg movement, trembling throughout the whole dance. Although it is leg movement, upper body rhythm is rich to balance the body and visual effect. The basic movements include swinging hands front and back to coordinate the lower body movement, and alternating right and left hands on the chest, which is more like the arm movements of Guozhuang dance and string dance, but with faster speed. Lower body movements can be summed up as: lift step, retreat step, bend leg quiver knee, tick step, leisurely step, slide step etc. According to the rhythm and content of the performance, the variety of dance is rich 。 Complementary with a few jump, turn round and rotate, the movements are clean and agile with bold style. In addition, the ankle of the dancers often with bells. Cheerful melody with clear bell makes the dance performance warm and hearty.

Tap dancing improves coordination, balance, and lower body strength. It is suitable for body turning movement, whole body movement and jumping movement of pupils.

With regard to Reba dance, compared with the above three dances, the range of movements of Reba dance is larger and the movements are more difficult. With the help of tambourine props, the rhythm is more rapid, cheerful scene. The movements of men and women, the leader and the group of Reba dance are different according to its span of difficulty. For example, some movements, such as low back and jump, are close to the requirements of acrobatics [4], all of which are performed by some dancers with superior abilities alone. 
Therefore, it is often considered to be a combination of dance, drama, sports and acrobatics. To sum up, although Reba dance is a very effective way for flexible training and increase of heart rate, it is not suitable to be used in the characteristic between-class exercise of pupils due to the safety consideration of the development of between-class exercise.

By summarizing and refining the movements of the above Tibetan dance types, we can select and use the movements symbolized by Tibetan dance, such as: front and back steps, knee flutter, front hand stroke, clap, outstretched hand gesture, ipsilateral body turn, etc., to replace the movements of the same exercise purpose, with Tibetan characteristic music melody, to achieve the basic requirements of characteristic between-class exercise.

\section{ARRANGEMENT ANALYSIS}

\section{A. Movement elements}

The most important characteristic of the Tibetan betweenclass exercise is that the exercise is different from the traditional "horizontal and vertical" waving and stepping, and uses the Tibetan symbolic movements instead. Therefore, based on the above analysis of the movements of traditional folk dance and the investigation of the teaching objects, we make the following arrangements for the movements of each section of this characteristic between-class exercise:

Stretching. The forward and backward steps in Guozhuang dance, combined with hand-clapping, knee-shaking and head movement on the same side, gradually transiting from small amplitude movement to arm exhibition movement.

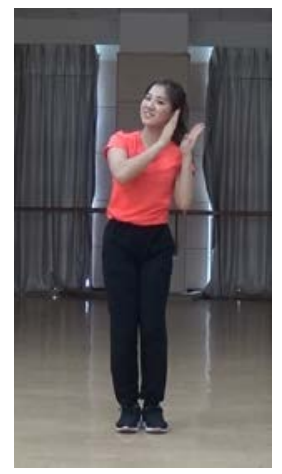

Fig. 1. Knee trembling + hand clapping

Chest expansion. Rowing by turns in string dance and repeat the rhythm of clapping to do chest expansions exercise. The lower body coordinates with the front and back steps to realize the coordination training of the same hand and foot in Tibetan dance.

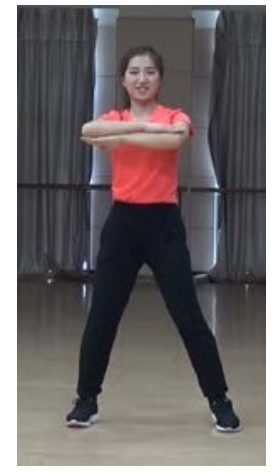

Fig. 2. Chest expansion preparation posture ${ }^{1}$

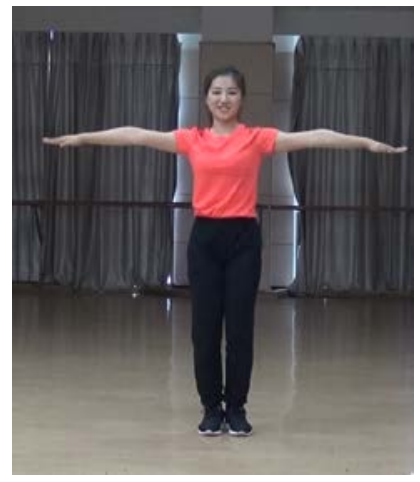

Fig. 3. Chest expansion posture

Kicking movement: prepare the kicking movement with stepping and knee trembling, and stepping forward and backward, and then kicking with legs in place, with waist slightly to the side of the kick. Clapping hands while kicking and rowing the same hands while stepping forward and backward.

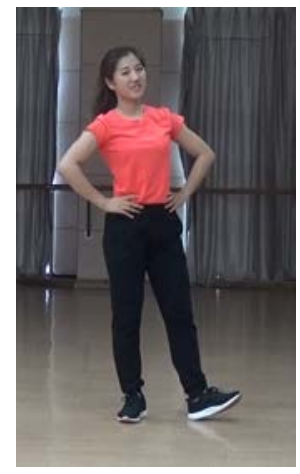

Fig. 4. Kicking preparation posture

\footnotetext{
${ }^{1}$ The image copyright belongs to the author and images are selected from videos in fund project of the article.
} 


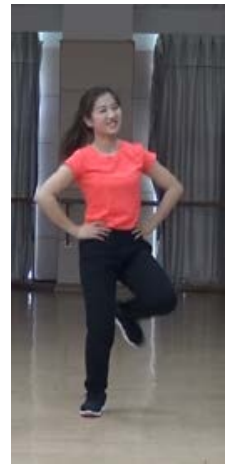

Fig. 5. Kicking movement

Side movement: extracting "three steps with one side leaning" in string dance with one hand raising, and then stretching and waving hands on both sides of the body, while turning the waist.

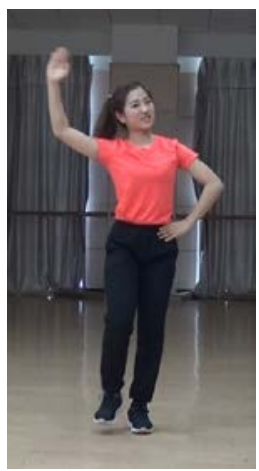

Fig. 6. Side movement

Turning movement: adopting the turning movement from Zhenda Guozhuang dance, bending slightly, bending the knee, lower one hand and raising the other and turning the body with the rhythm and then connecting the reverse position with knee trembling, changing hands, turning the body and then rotating.

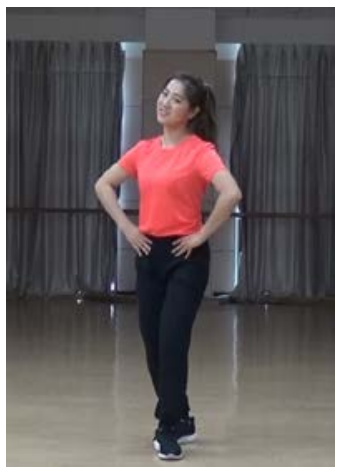

Fig. 7. Turning movement

General movement: stepping in place and the rhythm of hands gradually changes from clapping to stretching in turn, and the feet cooperate with the back-and-forth sliding step, which is similar to the action of presenting Hada in Tibetan areas, so as to achieve the purpose of coordinating the whole body movement.

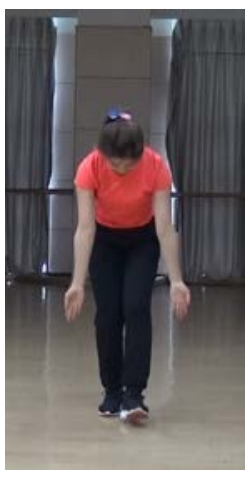

Fig. 8. Raising sleeves

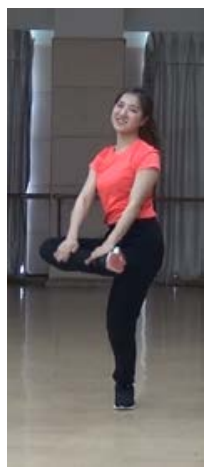

Fig. 9. Clapping legs

Arrangement movement: the jumping movement is replaced by the in-situ marking, and the range of movement is gradually reduced by the stretching of the hands. Finally, the rhythm of the movement in the stretching movement is returned with the rowing and stepping forward and backward.

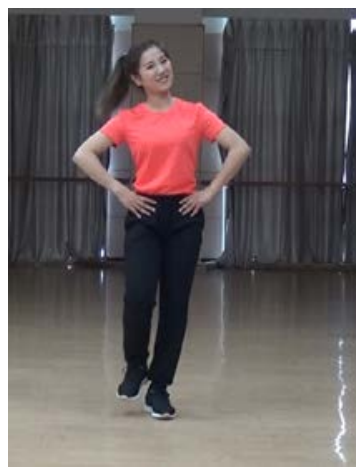

Fig. 10. Basic stepping

\section{B. Music Selection}

By comparing different styles of music, such as Guozhuang, string and tap dance, music of string style was chosen as the unified rhythm in between-class exercise. In general, string music in the middle of Guozhuang and tap in terms of style and speed. The music of between-class exercise adopts the string music with brisk rhythm and lively melody, which meets the aesthetic requirements of pupils. In the process of music allocation, we combine the movements in accordance with the overall structure and exercise sequence of the characteristic between-class exercises, and adjust the time, direction, 
connection and beat of the combination, so that music can play the best effect of command and assistance to the rhythm of the movements, so as to improve the fun of the whole set of characteristic between-class exercises and make the athletes happy. Meanwhile, in the long-term implementation process of characteristic between-class exercise, music and the coordination of movements are deeply rooted in people's hearts, laying a solid foundation for cultivating students' basic knowledge of Tibetan dance combination.

According to the Tibetan dance elements in the characteristic between-class exercise, the string music carries repeatability, which not only conforms to the characteristics of folk collective Tibetan dance, but also reflects the repetition, symmetry and regular changes in the arrangement. Among a variety of versions, the string music was determined by the Tibetan voice singing accompanied by string and flute respectively with brisk and regular rhythm and beautiful melody, reflecting distinct Tibetan culture characteristics.

\section{Section arrangements}

After extracting the exercise movements from the traditional dances in Tibetan areas, adjust the training intensity and schedule according to the overall arrangement of the school's activities between classes. Four eight-beat preparation and arrangement exercises are added into the whole characteristic exercise, which is used to arrange the formation and adjust the physical state, which is good for the connection activities between classes.

Next, each group of movements is arranged according to two eight-beat movements. First, the stretching exercise, which includes trembling steps with clapping hands and retreating with waving hands; secondly, chest expansion exercise, which includes stepping with rowing hands; thirdly, kicking movement, which includes trembling knees with clapping hands and lifting legs and sleeves; fourthly, side movement, which includes formation changes with four pupils as a group, forming a small flower with four petals. The formation changes by small jumps and extension and then raising sleeves, forwarding and retreating with steps which rowing hands and sliding the same feet; fifthly, turning movement, which includes stretching, twisting, waisting, bending knees and turning body as well as hands on hips and trembling knees; sixthly, general movement. The four-man formation changes back to horizontal and vertical alignment, which includes stepping back on the sleeves and forward and backward; seventhly, jumping, which includes raising sleeves with jumping and forwarding and retreating with steps. The eighth section is arrangement movement, which includes stretching and stepping, clapping hands, hands on hips and trembling steps.

Through the specific combination of the above sections, it can be seen that the overall arrangement of characteristic between-class exercises attaches great importance to the principle of repetition and symmetry of movements. For pupils, repetition can reduce learning difficulty, improve learning interest and ensure learning quality. Through repetition, the rules are established, the changes derived and the whole system is built, which is the key to make the whole set of features easy to learn and interesting [5]. In addition, symmetry of combined movements is not only one of the basic principles of the development of middle and primary school exercise, but also can meet the requirements of balanced exercise and development from the perspective of mechanics.

The characteristic between-class exercises arranged in the above way can achieve the training rhythm from the whole to the part and back to the whole again. The intensity of the exercise gradually increases and the exercise time meets the physical and mental development needs of pupils. To relieve the fatigue of classroom teaching, it is suitable for all teachers and students to practice together.

At present, between-class activities are gradually "popular" in primary and middle school around the country. The characteristic between-class exercise is no longer a single broadcast gymnastics, the pupils dance along with the brisk rhythm of activities instead. The introduction of characteristic elements makes the between-class exercise activities more fashionable and welcomed by pupils. The students rushed into the playground with great interest, looking forward to stretching their muscles and relaxing their mood through characteristic between-class exercises. Characteristic betweenclass exercise combines music dance major with social sports to break through the habitual thinking mode in the development of between-class exercise. Under the background of inheriting folk songs and dances in Ganzi Tibetan area, this activity is not only between-class dance but also make the exercise more like dance. The music dance with Tibetan characteristics in Ganzi was purposefully and directionally selected and added to the between-class exercise of primary school students, so that pupils can get in touch with traditional music and dance every day in campus life, understand and be familiar with these traditional music and dance forms, achieving the purpose of inheriting Ganzi folk music and dance. At the same time, it also creates the characteristic campus culture of the school.

\section{REFERENCES}

[1] Chen Panpan. The Compilation and Creation of Between-Class Exercises with Tibetan Characteristics [C]. Chengdu: Chengdu Sports University Department of Physical Education, 2016.6

[2] Chen Yingwu. On the Generation, Present Situation and Development of Between-Class Exercise in Primary and Secondary Schools in China [C] Jiangxi: Sports College of Jiangxi Normal University, 2004.5

[3] Mu Yang. The Characteristics of Tibetan Dance in Ganzi Seen from the Material of "Impression Tangka” [J]. Jilin: Modern Music, 2016.5

[4] Cai Chuan. Reba: A Kangba Tibetan Dance [J]. Hebei: Dawutai, 2013.10

[5] Zhang Xianghua. On Teaching Characteristics and Fitness Value of Tibetan Guozhuang Dance [J] Inner Mongolia: Journal of Inner Mongolia Normal University, 2014.8 